\title{
Pratiques religieuses et lieux de culte partagés entre islam et christianisme (autour de la Méditerranée)
}

À propos de :ALBERA Dionigi, COUROUCLI Maria, (dirs.), Religions

traversées. Lieux saints partagés entre chrétiens, musulmans et juifs en Méditerranée, Arles, Actes Sud, 2009, 359 p.VIVIER-MUREşAN Anne-Sophie, (dir.), "Coexistence et conflits communautaires en Méditerranée ", Chronos, 18, 2008, 265 p.AUBIN-BOLTANSKI Emma, « La Vierge, les chrétiens, les musulmans et la nation. Liban, 2004-2007 », Terrain, 51, 2008, pp. 10-29.

\section{Bernard Heyberger}

\section{(2) OpenEdition}

\section{Édition électronique}

URL : http://journals.openedition.org/assr/21994

DOI : $10.4000 /$ assr.21994

ISSN : $1777-5825$

Éditeur

Éditions de l'EHESS

\section{Édition imprimée}

Date de publication : 31 mars 2010

Pagination : $273-283$

ISBN : 978-2-7132-2253-5

ISSN : 0335-5985

Référence électronique

Bernard Heyberger, «Pratiques religieuses et lieux de culte partagés entre islam et christianisme (autour de la Méditerranée) », Archives de sciences sociales des religions [En ligne], 149 | janvier-mars 2010, mis en ligne le 15 novembre 2012, consulté le 30 avril 2019. URL : http:// journals.openedition.org/assr/21994; DOI : 10.4000/assr.21994 


\title{
Bernard Heyberger
}

\section{Pratiques religieuses et lieux de culte partagés entre islam et christianisme (autour de la Méditerranée)}

\author{
À propos de : \\ Albera Dionigi, Couroucl Maria, (dirs.), Religions traversées. Lieux \\ saints partagés entre chrétiens, musulmans et juifs en Méditerranée, \\ Arles, Actes Sud, 2009, 359 p. \\ VIVIER-MureŞAn Anne-Sophie, (dir.), " Coexistence et conflits commu- \\ nautaires en Méditerranée », Chronos, 18, 2008, 265 p. \\ Aubin-Boltanski Emma, «La Vierge, les chrétiens, les musulmans et la \\ nation. Liban, 2004-2007 », Terrain, 51, 2008, pp. 10-29.
}

Ce n'est sans doute pas simple coïncidence si ces trois publications ont paru à peu de semaines de distance. Plusieurs auteurs signent d'ailleurs un article dans chacun des deux ouvrages collectifs. Leur sujet principal est le même, et répond à une interrogation actuelle : celui des formes de contacts, conflictuels, concurrentiels ou pacifiques, dans la pratique religieuse, entre les adeptes de l'islam et ceux du christianisme dans l'espace méditerranéen, parfois conçu de manière extensive (un article sur l'Iran et un sur la Hongrie dans le numéro de Chronos).

On sait gré aux maîtres d'œuvre de ne jamais envisager une « aire culturelle », voire une "identité » méditerranéennes, mais de considérer simplement les abords de la Mer intérieure comme un terrain d'observation privilégié des processus qu'ils tentent d'analyser. Il est aussi particulièrement stimulant de traiter ensemble du Proche-Orient et de la région des Balkans. Dans les deux cas, on assiste à des formes de renouveau religieux faisant des sanctuaires et lieux de pèlerinages des enjeux pour l'occupation de l'espace public, et donc pour les luttes politiques, dans un contexte assez éloigné de celui qui nous est familier en Europe de l'Ouest. Plusieurs textes mettent en lumière l'importance de la phase de construction des États nationaux ( $\mathrm{XIX}^{\mathrm{e}}$ et $\mathrm{XX}^{\mathrm{e}}$ siècles), pour comprendre les 
situations actuelles ${ }^{1}$. S'il est vrai que conflit et convivialité autour de ces territoires relèvent des mêmes processus, et que le «critère ethnique ou national prime nettement sur le religieux et s'y substitue presque ${ }^{2}$, plusieurs articles démontrent néanmoins que la distinction entre la sphère politique et la sphère religieuse n'est pas évidente à établir, et que les enjeux confessionnels internes à chaque communauté jouent un rôle central dans la nature de la relation avec l'autre.

Seul Henk Driessen traite du contact entre juifs et musulmans dans le volume Religions traversées ${ }^{3}$. Galia Valtchinova, dans ses contributions aux deux volumes ${ }^{4}$, et Anna Poujeau dans Religions traversées ${ }^{5}$ ont le mérite de rappeler que la question du contact entre christianisme et islam se double très souvent de celle de la coexistence de formes différentes de christianisme et d'Églises sur le même territoire. Dans Chronos, Catherine Mayeur-Jaouen ${ }^{6}$ évoque l'essor d'un pèlerinage à Kom Gharîb dans un coin retiré de Haute-Égypte où la guérilla islamiste a pris les chrétiens pour cible entre 1992 et 2002, comme " une revanche " des coptes-orthodoxes sur les coptes-catholiques, de plus en plus minoritaires dans cette région. Dans le même volume, l'article de Filippo M. Zerilli traite de la rivalité entre les membres de l'Église orthodoxe roumaine et la minorité appartenant à l'Église gréco-catholique, dans un village de Transylvanie ${ }^{7}$, et celui de Gheorghe Şişeştean de la situation de ces deux minorités dans la Hongrie majoritairement catholique romaine ${ }^{8}$, depuis la chute du communisme. Enfin, l'article d'une très grande profondeur historique que Dan Ioan Mureşan consacre à Jérusalem évoque la captation successive de l'imaginaire de la Ville Sainte par les trois religions, et la rivalité exacerbée que les Lieux Saints chrétiens génèrent entre dénominations différentes, jusqu'à nos jours ${ }^{9}$.

1. Anastassiadou, 2005 : cet ouvrage, qui s'intéresse moins aux aspects strictement religieux ou personnels de l'identité minoritaire (culte, croyances, mariages, funérailles, instruction, recours à la justice du cadi, paiement de la capitation...) qu'au rapport entre celle-ci et l'espace urbain, donne plusieurs contributions intéressantes sur cette question à l'ère des nationalismes.

2. Introduction d'Anne-Sophie Vivier-Mureşan, « Coexistences et conflits communautaires en Méditerranée : l'enjeu des sanctuaires et lieux de culte », Chronos, p. 9.

3. «Un sanctuaire juif et musulman dans le Nord du Maroc : échos d'un passé ambigu ", pp. 209-217.

4. «Le Mont de la Croix : partage et construction des frontières dans un lieu de pèlerinage bulgare ", dans Religions traversées, pp. 113-140 et " "Jérusalem des Rhodopes” vs. "La Mecque des Rhodopes” deux lieux de pèlerinage entre la Bulgarie, la Grèce et la Turquie " Chronos, pp. 55-86.

5. «Partager la baraka des saints. Des visites pluriconfessionnelles aux monastères chrétiens de Syrie ", dans Religions traversées, pp. 295-319.

6. "Abûnâ Yassâ : un pèlerinage copte-orthodoxe face aux catholiques et aux musulmans ", Chronos, pp. 109-128.

7. «Néolibéralisme, identité locale et politique de la foi. La construction d'une nouvelle église gréco-catholique à Mihalt (Roumanie) », Chronos, pp. 87-105.

8. "Orthodoxes et gréco-catholiques roumains de la Hongrie entre constructions et reconstructions identitaires ", Chronos, pp. 189-207.

9. "Aux prises avec le centre du monde, autour du double paradoxe des "lieux saints chrétiens" de Jérusalem ", Chronos, pp. 29-54. 
Le pluralisme musulman pour sa part n'apparaît qu'en arrière-fond dans les articles. Il est cependant évident que le partage de la perception et de la pratique du sacré est plus facile et plus généralisé avec des dissidents de l'islam (druzes, nusayris, bektachis, alévis, ahl ul-haqq) qu'avec les tenants de l'islam sunnite. C'est dans ces courants qu'on trouverait une véritable volonté et une véritable pensée pour atteindre une synthèse religieuse qu'on peut qualifier de "syncrétisme ", alors que ce qui est généralement présenté dans les contributions réunies ici concerne surtout la mixité et la porosité des pratiques, fondées sur un certain flou doctrinal, bien éloignées d'un véritable «métissage " ${ }^{10}$.

Si l'on peut repérer le cas de chrétiens visitant des sanctuaires musulmans (en Macédoine, en Égypte) ${ }^{11}$, l'inverse est beaucoup plus fréquent : les musulmans sont bien plus nombreux à se rendre en dévotion dans des lieux de culte chrétiens. Dans l'apologétique chrétienne, il est très courant, depuis longtemps, de donner des musulmans comme principaux garants d'un fait miraculeux, attestant la vérité du christianisme : c'est " la preuve par le musulman », qui permet, grâce au miracle, d'inspirer un sentiment de supériorité à celui qui n'a pas le pouvoir et la force de son côté ${ }^{12}$. Ainsi, ce sont des ouvriers musulmans qui ont été les premiers à apercevoir la Vierge apparue au-dessus de la chapelle de Zaytûn dans la banlieue du Caire en $1968^{13}$, et c'est un enfant jordanien, appelé Muhammad, qui fut le premier témoin des miracles de la Vierge de Béchouate en $2004^{14}$. Un autre argument de l'apologétique chrétienne, qu'on rencontre en Albanie, consiste à présenter les musulmans comme des crypto-chrétiens convertis à l'islam ${ }^{15}$. Par ailleurs, l'islam prétend se situer dans la continuité du judaïsme et du christianisme, et en intégrer, ou absorber, le message religieux. Dans le Coran, le hadith, ou les qisas al-anbiy $\bar{a}$, pour lesquels les récits des évangiles canoniques et apocryphes ont servi de source, Marie et Jésus occupent une place exceptionnelle ${ }^{16}$. Le saint cavalier et martyr Georges (à présent rayé du synaxaire catholique pour cause d'inauthenticité), très populaire chez les chrétiens orientaux, est entré dans la religion musulmane après l'implantation des Arabes en Palestine au $\mathrm{VII}^{\mathrm{e}}$ siècle, qui l'ont assimilé à la figure légendaire d'al-Khidr. Il est parfois associé à saint Élie ${ }^{17}$. Il est vénéré comme Hidrellez chez les Turcs d'Anatolie ${ }^{18}$.

10. Sur le syncrétisme, Glenn Bowman, «Processus identitaires autour de quelques sanctuaires partagés en Palestine et en Macédoine », dans Religions traversées, pp. 27-52 ; Bojan Baskar, "S'occuper du sanctuaire du voisin en Bosnie-Herzégovine ", ibid., pp. 85-112 ; Dionigi Albera, "Conclusion ", ibid., p. 322. Sur ce sujet, B. Heyberger, R. Madinier, 2010.

11. G. Bowman, op. cit.; C. Mayeur-Jaouen, " "Que partagent les coptes et les musulmans d'Egypte ?" L'enjeu des pèlerinages ", dans Religions traversées, pp. 219-254.

12. C. Mayeur-Jaouen, op. cit. (Chronos).

13. Sandrine Keriakos, "Les apparitions de la Vierge en Égypte: un lieu privilégié de la rencontre entre coptes et musulmans ? ", dans Religions traversées, pp. 255-294.

14. E. Aubin-Boltanski, op. cit.

15. Gilles de Rapper, "Vakëf: lieux partagés du religieux en Albanie ", dans Religions traversées, pp. 53-83.

16. E. Aubin-Boltanski, op. cit.; S. Keriakos, op. cit.

17. G. Bowman, op. cit.

18. Maria Couroucli, "Saint Georges l'Anatolien, maître des frontières ", dans Religions traversées, pp. 175-208. 
On a tendance de nos jours à interpréter positivement, dans une perspective de « dialogue islamo-chrétien » et d'éloge de la « tolérance » cette proximité et cette indétermination : le sanctuaire de Béchouate, conçu comme un lieu de résistance maronite pendant la guerre des milices (1975-1990), est aujourd'hui présenté par les autorités ecclésiastiques, inspirées du Vatican, comme un lieu de rencontre $^{19}$. De même, le pèlerinage à saint Antoine de Padoue, dans le nord de l'Albanie, est une occasion pour les responsables des différentes confessions d'affirmer leur bonne entente ${ }^{20}$. En Bosnie et en Bulgarie, la notion de komšiluk/ komşuluk, désignant une pratique de bon voisinage, de tolérance multi-ethnique et de cohabitation pacifique héritée des Ottomans, a été mise en valeur par les ethnologues et est devenue aujourd'hui centrale dans le discours " politiquement correct ${ }^{21}$. Mais la confusion religieuse entretenue par l'islam n'est pas sans danger pour les minoritaires, du point de vue des autorités ecclésiastiques, qui, très tôt, ont développé des argumentaires apologétiques et didactiques pour prévenir leurs ouailles d'une interprétation islamique du message chrétien ${ }^{22}$. La peur d'une captation islamique d'un de leurs sanctuaires se vérifie chez les Arméniens de Fereydân (Iran), une région devenue musulmane depuis l'exil des ruraux chrétiens vers Ispahan ou l'étranger. Le discours recueilli par l'enquêtrice, auprès du voisinage musulman du tombeau du saint martyr présumé, livre en effet des indices sur ce processus insidieux d'appropriation symbolique et d'islamisation du lieu ${ }^{23}$.

Les deux ouvrages collectifs se réfèrent abondamment au travail pionnier de Frederick Hasluck ${ }^{24}$, auxquels ils rendent hommage. Le savant britannique adhérait à l'hypothèse que le partage des sanctuaires avait conduit à la progressive mainmise musulmane. Mais il a eu le mérite de mener une vaste enquête historique et anthropologique sur le sujet à la veille de la Première Guerre mondiale, alors que les phénomènes étudiés ici étaient encore beaucoup plus répandus qu'aujourd'hui, et s'inscrivaient dans une très longue durée. Les nationalismes, le communisme, la disparition des sociétés rurales, et ce que l'on pourrait qualifier de "confessionnalisation ", n'avaient alors pas encore produit tous leurs effets.

Les auteurs se sont tous efforcés à juste titre de contextualiser leur enquête, ce qui rend la synthèse comparative difficile. Il n'en ressort pas moins un certain nombre de traits que l'on peut tenter de résumer.

Le premier, c'est l'inscription de ces pratiques communes dans un territoire précis, généralement partagé. Mais ce partage n'implique pas forcément une égalité entre les partenaires : au contraire, c'est l'inégalité qui favorise, voire explique,

19. E. Aubin-Boltanski, op. cit.

20. G. de Rapper, op. cit.

21. B. Baskar, op. cit.; G. Valtchinova, art. cit. (dans Religions traversées).

22. Griffith, 2008.

23. Anne-Sophie Vivier-Mureşan, "Minorités en partance et lieux de culte partagés. L'exemple des Arméniens d'Iran ", dans Chronos, pp. 169-187.

24. Hasluck, 1929. 
les pratiques de "partage compétitif » ${ }^{25}$, qui sont aussi des moments de mimétisme ou de rivalité. Les chrétiens qui accueillent les musulmans sur leur territoire sacré sont en général en situation d'infériorité, et leurs sanctuaires ont un caractère de refuge ou d'enclave, à l'écart des villes ou dans un quartier spécifique, là où le sacré chrétien peut se déployer avec plus de liberté, et où les chrétiens peuvent se retrouver entre eux.

On peut adopter la distinction opérée à juste titre par A.-S. Vivier-Mureşan, entre « lieu de culte » et "sanctuaire " ${ }^{26}$. Le premier est destiné à accueillir les actes liturgiques réguliers, tandis que le second se définit comme lieu de pèlerinage individuel ou collectif, jouissant d'un charisme particulier, autour d'une figure sainte. Généralement, ces sanctuaires sont sur des marges, matériellement situées dans une zone de confins, où différentes ethnies et confessions se touchent (comme c'est le cas à Béchouate au Liban, ou à Kraštova Gora, le Mont de la Croix, en Bulgarie) mais aussi symboliquement à l'écart du monde, comme le monastère de sainte Thècle à Ma'lûlâ en Syrie. Comme le note justement D. Mureşan à propos de Jérusalem, "c'est la séparation entre [le] centre politique et le centre spirituel, avec de surcroît l'éloignement qui donne au voyage sa signification de purification à travers un effort volontaire, pour retrouver la vérité originelle dont le quotidien semble s'éloigner ". Et " la périphérisation d'un centre par rapport à tous le rend un centre pour tous : le centre de pèlerinage se doit d'être essentiellement périphérique, sa périphéricité géographique et culturelle devenant la condition même de sa centralité spirituelle " ${ }^{27}$. Dans cette perspective, la volonté de faire de Jérusalem un centre politique - la capitale de l'État d'Israël - est problématique.

Ces lieux sont ancrés dans un paysage précis, et relèvent souvent d'une sacralité chtonienne, définie par la présence du rocher, de la grotte, de la source, du cénotaphe du saint, éléments d'un sacral presque universel ${ }^{28}$, identifiable et acceptable pour les membres de diverses dénominations religieuses. Dans l'Albanie postcommuniste, le terme de vakëf, désignant les fondations pieuses en droit islamique, a perdu son sens institutionnel pour nommer "un lieu sacré doté de certains pouvoirs, qui survit à la disparition du lieu de culte institutionnel ». Il s'applique à « des lieux qui sont soit périphériques par rapport au lieu de culte principal du village, église ou mosquée, soit abandonnés en tant que lieu de culte principal. Ce sont des lieux d'où une certaine forme d'activité religieuse s'est retirée ${ }^{29}$. Très souvent les sanctuaires et leur saint ont été au centre d'un

25. L'expression " competitive sharing » est de Robert M. Hayden, dont les articles sont abondamment cités et discutés par les contributeurs. Voir Hayden, 2002.

26. A.-S. Vivier-Mureşan, "introduction ", op. cit., p. 12.

27. D. Mureşan, op. cit., p. 38. L'auteur suit Victor Turner, une référence fréquente dans les contributions à ces deux volumes : Turner, 1973 ; Turner et Turner, 1978.

28. Dupront, 1978.

29. G. de Rapper, op. cit. 
calendrier agro-liturgique que les ruraux d'un même terroir partageaient pour l'essentiel : les fêtes de saint Georges et de saint Dimitri divisaient l'année en deux saisons ${ }^{30}$, et le mouled de saint Michel en Haute-Égypte coïncidait avec le début de l'inondation avant la régulation du $\mathrm{Nil}^{31}$.

Alors que le pèlerin imagine trouver la permanence, la fidélité, l'ancrage, la stabilité, dans ces lieux qu'il visite pieusement, le fait est que, soit ils ont une histoire longue mais chaotique faite de reculs et de restaurations, soit ils sont de création plutôt récente, mais revendiquant une tradition réinventée. Ce sont en tout cas des " lieux cristallisateurs de la mémoire collective » ${ }^{32}$, et où des conflits de mémoires surgissent ou s'apaisent. Des lieux de l'origine rêvée, comme Jérusalem, bien sûr. Des lieux «berceaux », qui, dans les Balkans, se situent généralement à la périphérie des espaces nationaux ${ }^{33}$. À Genazzano, près de Rome, l'histoire du transfert miraculeux d'une icône de la Madonna del Buon Consiglio de Scuttari (Albanie) vers ce bourg du Latium lors de la conquête turque de l'Albanie, en 1467, est racontée suivant différentes modalités discursives, réinterprétant la mémoire du passé en fonction des relations actuelles entre Italiens et Albanais depuis la chute du communisme ${ }^{34}$. Le séjour de la Vierge et de la Sainte Famille en Égypte donnerait aux coptes, qui multiplient les lieux présumés de ce séjour au fur et à mesure que leur appartenance à la nation égyptienne est mise en cause, un privilège d'ancienneté et d'autochtonéité sur les musulmans ${ }^{35}$. SaintGeorges, sur l'île de Buyuk Ada, près d'Istanbul, après avoir été le sanctuaire des Grecs de la ville, devient aujourd'hui un lieu de mémoire des musulmans de la métropole, qui cherchent dans une survivance de la tolérance ottomane une alternative au modèle national homogène turc ${ }^{36}$. Pour les communautés en diaspora, déracinées de leur terroir, un sanctuaire peut offrir un point de ralliement et de fixation de la mémoire, un moyen de recréer, le temps d'un pèlerinage, une communitas, tout en s'affirmant contre un voisinage hostile et dominateur. C'est le cas des pèlerins arméniens sur le tombeau de Lisangis, à Ferdeyân, et des musulmans turcs ou pomaks bulgarophones à Yenihan baba tekke, "La Mecque des Rhodopes », faisant face à Kraštova Gora, la « Jérusalem des Rhodopes » 37.

30. M. Couroucli, op. cit.

31. C. Mayeur-Jaouen, op. cit., dans Religions traversées.

32. Selon la formule de Maurice Halbwachs (1950), citée par A.-S. Vivier-Mureşan, " introduction ", op. cit., pp. 14-15.

33. G. Valtchinova, op. cit. (Chronos).

34. Vincenzo Cannada Bartoli, «Le mythe partagé. Produire l'histoire, chercher mémoire à Genazzano (Rome) ", Chronos, pp. 231-256. L'histoire de ce transfert susciterait la comparaison avec celui de Lorette (de la Croatie vers les Marches), vers la même époque. Mais Lorette a eu un autre destin, devenant le sanctuaire national italien : Scaraffia, 1998.

35. S. Keriakos, op. cit. Voir aussi sur ce sujet Lucette Valensi, La Fuite en Égypte, Histoires d'Orient et d'Occident, Paris, Seuil, 2002.

36. M. Couroucli, op. cit.

37. A.-S. Vivier- Mureşan, "Minorités en partance... ", op. cit.; G. Valtchinova, op. cit. (dans Religions traverses; Chronos). 
Plus étonnant est le cas des membres de la diaspora pakistanaise en Europe, adeptes d'une organisation, le Minhaj ul-Quran, constituant une « communauté imaginée et déterritorialisée » autour d'un fondateur charismatique, Tahir ul-Qadri, qui s'en vont, sous la houlette de celui-ci, se ressourcer aux sanctuaires des grands "saints » soufis du Proche-Orient, Rumi à Konya, Abraham Bin Adham à Jebblé, Ibn Arabi à Damas, mais aussi des conquérants (Saladin) et des " saintes » chiites Zainab et Ruqaya dans cette dernière ville ${ }^{38}$.

Ces lieux offrent aussi la possibilité à une personnalité charismatique d'exercer une influence en marge de sa propre appartenance religieuse, et de susciter une dévotion qui sort des cadres institutionnels. On en trouve mention dans l'histoire de Béchouate, de Kraštova Gora, ou de Kôm Gharîb. C’est également le cas des religieuses de Ma'lûlâ, en contact direct avec leur sainte, ce qui leur confère une forme de charisme contestataire et le pouvoir de transmettre la baraka aux pèlerins. La figure du " saint vivant ", dont les grâces pouvaient inspirer la vénération des chrétiens et des musulmans, attestée dans le passé, n'apparaît guère dans les contributions réunies ici. Elle n'est mentionnée que pour l'Égypte, avec les saints coptes récents Cyrille VI, Umm Irénè (morte en 2006), Abûnâ Yassâ (mort en 1962), ou le cheikh soufi Ahmad Radwân (mort en 1967). Jean-Paul II a pu jouir de ce type d'aura chez certains musulmans. Tahir ul-Qadri incarne le « saint vivant ", mais uniquement pour ses adeptes musulmans. Les franciscains, desservants de l'église Saint-Antoine-de-Padoue dans une artère principale d'Istanbul ${ }^{39}$, sans être dotés d'un charisme particulier, sont néanmoins depuis longtemps des experts du contact avec l'islam, associant le culte du martyre à la négociation permanente avec les musulmans. Ils sont aujourd'hui, en particulier, porteurs de « l'esprit d'Assise » insufflé par Jean-Paul II à partir de la rencontre de 1986.

Ce qui paraît rapprocher les fidèles des deux religions qui fréquentent ces lieux, c'est une certaine communauté du geste pieux. Les contributions livrent de nombreux exemples de ces pratiques. Celles-ci peuvent parfois s'interpréter en termes d'influence réciproque : ainsi, le fait que des chrétiens puissent considérer la consommation de vin ou de porc comme harām ou que les musulmans adoptent l'usage catholique de l'image pieuse pour leurs propres saints et cheikhs ${ }^{40}$.

Mais, plus généralement, le comportement pèlerin s'ancre dans une pratique presque immémoriale et universelle. Font partie de cette structure rituelle l'ascension au sanctuaire à pied, au moins sur les derniers mètres, la circumambulation autour d'un cénotaphe, le toucher d'une pierre aux vertus propitiatoires, la

38. Alix Philippon, "Quand la communauté n'est plus seulement imaginée... », Chronos, p. 209-229.

39. Dionigi Albera, Benoît Fliche, «Les pratiques dévotionnelles des musulmans dans les sanctuaires chrétiens : le cas d'Istanbul ", dans Religions traversées, pp. 141-174. Voir aussi l'allusion aux franciscains de Bosnie dans B. Baskar, op. cit., et à ceux de Jérusalem dans D. Mureşan, op. cit.

40. C. Mayeur-Jaouen, op. cit., dans Religions traversées; Mayeur-Jaouen, 2003. 
consommation d'eau, la combustion de cierges ou d'encens, l'offrande d'argent ou de nourriture, l'attachement d'un ruban à une grille ou à un arbre. Les gestes mêmes de la prière : se déchausser à l'entrée du sanctuaire, tendre les bras, paumes tournées vers le haut, se prosterner en pratiquant des " métanies ", qui aujourd'hui nous évoquent plutôt la piété musulmane, sont en fait les façons traditionnelles de prier en Orient. Dormir auprès du saint, faire des ablutions avec l'eau, emporter chez soi un coton imbibé d'huile, voire une portion de la terre du sanctuaire qu'on peut boire en potion mélangée à de l'eau, sont aussi des gestes communs et anciens. L'incubation, qui consiste à passer une nuit dans un sanctuaire pour obtenir la guérison du saint, celui-ci apparaissant alors généralement en songe, est attestée depuis l'Antiquité ${ }^{41}$. Le baptême prophylactique demandé par les musulmans pour leurs enfants a été longtemps chose très commune ${ }^{42}$. Certaines pratiques, mentionnées dans les récits des contributeurs, sont peut-être en voie de disparition, comme le fait de nouer un long ruban avec lequel on entoure le territoire du sanctuaire ${ }^{43}$. Le kourban, le sacrifice sanglant d'un animal accompagné de sa consommation en pique-nique près du sanctuaire, donné de nos jours dans les Rhodopes comme typiquement musulman ${ }^{44}$, a été également, et est encore parfois, une pratique chrétienne ${ }^{45}$. Tout ceci est révélateur d'une culture commune, reposant sur une représentation commune du sacré. Dans celle-ci, les visions pendant le sommeil occupent une place importance. La Vierge ou le saint annoncent en rêve une guérison, ou prescrivent une dévotion, ou menacent de vengeance celui qui aurait attenté à leur sanctuaire ou à leur image ${ }^{46}$. Cette injonction reçue en songe relève d'un autre type d'autorité que l'autorité institutionnelle, et permet de transcender les différences confessionnelles et de contourner des obstacles institutionnels.

Mais le partage apparent d'une même culture religieuse n'implique pas forcément adhésion entière au même système de croyance. Le baptême, pour le chrétien ordinaire comme pour le musulman, est censé apporter à l'enfant qui le reçoit une forme de protection contre le mauvais œil. Mais il revêt un sens supplémentaire pour un chrétien, puisqu'il s'agit d'un sacrement par lequel un nouveau membre est reçu dans l'Église, communauté des croyants, et y reçoit une grâce spécifique. De même, la consommation de l'hostie consacrée, parfois recherchée par des musulmans, peut être interprétée comme propitiatoire ou prophylactique. Mais elle revêt un sens plus profond et plus riche pour un chrétien, à travers la théologie très élaborée de l'Eucharistie. Plusieurs articles relèvent que les musulmans prêtent aux religieux chrétien un pouvoir sur les esprits. Ils sont en particulier

41. Pratique relevée par G. de Rapper, op. cit. et par C. Mayeur-Jaouen, op. cit. (Chronos).

42. Pratique relevée par G. Bowman, op. cit., et par C. Mayeur-Jaouen, op. cit., dans Religions traversées.

43. G. Bowman, op. cit.

44. G. Valtchinova, op. cit. (dans Religions traversées et Chronos).

45. Mentionnée comme chrétienne par G. de Rapper, op. cit., elle est indiquée sans précision confessionnelle par A. Poujeau, op. cit.

46. S. Keriakos, op. cit.; G. De Rapper, op. cit.; A. Poujeau, op. cit. 
sollicités pour exorciser les possédés ${ }^{47}$. Il y a certes là une reconnaissance du sacré de l'autre, mais d'un sacré négatif, qui entretient des relations inquiétantes avec des forces maléfiques, confinant à la sorcellerie, et que le clergé chrétien aurait le pouvoir de lier et de délier. La croix, l'hostie, le volume des Évangiles ou la formule du prologue de l'évangile de saint Jean, dans ce contexte, peuvent être investis d'une puissance magique particulière ${ }^{48}$.

Si les gestes apparaissent souvent identiques, une observation attentive montre que les usages d'un lieu suivent des chronologies différentes selon les communautés, qui aboutissent à des différenciations marquées. La chapelle Saint-Nicolas à Makendoski Brod (Macédoine), un ancien türbe bektachi, est débarrassée de tout ce qui y témoigne de la fréquentation musulmane et pourrait y faire penser à une mosquée, lors de la préparation de la fête de saint Georges, pendant laquelle des centaines de visiteurs, presque exclusivement orthodoxes, y affluent ${ }^{49}$. À Istanbul, la fête de saint Georges (le 23 avril) a été instituée fête nationale turque (des enfants et de la République) ${ }^{50}$, ce qui permet à une foule considérable d'embarquer pour le monastère du saint à Buyuk Ada ce jour-là. Mais le calendrier liturgique chrétien ne coïncide pas toujours avec le calendrier national, et saint Georges peut être (assez souvent) fêté par les chrétiens grecs à une autre date que le 23 avril, donnant alors à la fête une coloration confessionnelle exclusive. À Istanbul encore, la fréquentation musulmane de Saint-Antoine-de-Padoue se concentre sur les mardis ${ }^{51}$. Et à Ferdeyân, la présence arménienne se cantonne au pèlerinage annuel, la semaine suivant Pâques, tandis que les musulmans (surtout les musulmanes) des environs peuvent se rendre quotidiennement sur la tombe du saint ${ }^{52}$.

Tous les auteurs s'accordent sur le fait que le sacré ne peut être partagé lorsque le contrôle clérical et institutionnel est renforcé. Un saint dont les contours restent flous est plus acceptable pour «l'autre " qu'un saint à la définition confessionnelle précise. L'action du clergé consiste très souvent à marquer l'identité religieuse d'un lieu, à y formaliser et à y encadrer la pratique. Il s'agit en particulier de préserver le cœur liturgique, la messe et les sacrements, de toute confusion ou malentendu en interdisant la communication avec l'autre au moment de ces rites. Les non-orthodoxes sont éconduits de la communion au pèlerinage de Kôm Gharîb. Les non-chrétiens n'accèdent qu'exceptionnellement à l'église du monastère de Sainte-Thècle de Ma'lûlâ, où les religieuses canalisent le flux

47. C. Mayeur-Jaouen, op. cit., dans Religions traversées. S. Kiriakos, op. cit. D. Albera, B. Fliche, op. cit. A.-S. Vivier-Mureşan, "Minorités en partance... ", op. cit.

48. G. Bowman, op. cit. C. Mayeur-Jaouen, op. cit. dans Religions traversées. S. Kiriakos, op. cit.

49. G. Bowman, op. cit.

50. M. Couroucli, op. cit. La République syrienne en a fait aussi un jour férié, consacré aux « martyrs".

51. D. Albera, B. Fliche, op. cit.

52. A.-S. Vivier-Mureşan, "Minorités en partance... ", op. cit. 
des musulmans exclusivement vers le sanctuaire de la sainte. L'action du clergé vise d'abord à séparer le sacré du profane, le religieux du superstitieux, chez ses propres ouailles, mais elle aboutit à introduire de la distinction et de la discrimination confessionnelle entre les dévots. À Jérusalem, les membres hellènes de la confrérie du Saint-Sépulcre pratiquèrent longtemps une politique d'affirmation de l'orthodoxie qui tenait à distance les musulmans et les chrétiens des autres rites, ou qui rejetait leurs propres fidèles palestiniens du côté de la « superstition des Arabes ", partagée selon eux par tous les autochtones ${ }^{53}$.

Beaucoup de situations évoquées dans ces ouvrages donnent l'impression que la mainmise ecclésiastique tend de nos jours à se renforcer, et à rendre plus difficiles les formes moins encadrées et donc plus syncrétiques de dévotion, au profit d'une homogénéisation. La notion de confessionnalisation, forgée par les historiens allemands de la Réforme et de la Contre-Réforme, aurait pu opportunément servir à éclairer certaines évolutions qui sont décrites ici. L'exacerbation de la concurrence entre confessions oblige chacune à définir plus précisément ses fondements dogmatiques et à mieux contrôler ses fidèles, ce qui aboutit au renforcement institutionnel et doctrinal, mais aussi à une rationalisation des comportements pour définir un genre de vie spécifique, caractérisé par des pratiques religieuses et des mœurs distinctives. Cette « confessionnalisation " concerne ainsi la société tout entière ; elle apparaît notamment directement connectée à la construction de l'État moderne ${ }^{54}$.

Si donc, dans l'église Saint-Antoine-de-Padoue d'Istanbul, l'espace est divisé entre la nef centrale, réservée aux offices et aux dévotions spécifiquement catholiques, et les nefs collatérales, où les fidèles de toutes confessions peuvent venir allumer un cierge devant la statue du saint ou simplement se promener en touristes, cette disposition n'a rien de particulier : elle ne fait que reproduire la discipline du Concile de Trente, à l'origine de la « confessionnalisation catholique ${ }^{55}$.

\section{Bernard HEYBERGER \\ École Pratique des Hautes Études - Université François-Rabelais (Tours) bernard.heyberger@wanadoo.fr}

53. D. Mureşan, op. cit.

54. Reinhard, 1995. W. Reinhard et H. Schilling sont les deux concepteurs de cette notion de Konfessionalisierung qui a produit une importante discussion historiographique, dans les années quatre-vingts.

55. D. Albera, B. Fliche, op. cit. 


\section{Bibliographie}

ANASTASSIADOU Méropi (éd.), 2005, "Identités confessionnelles et espace urbain en terres d'islam », numéro thématique de Revue des Mondes Musulmans et de la Méditerranée, 107-110, $544 \mathrm{p}$.

Dupront Alphonse, 1987, Du sacré. Croisades et pèlerinages. Images et langages, Paris, Gallimard.

GrIFFITH Sidney H., 2008, The Church in the Shadow of the Mosque. Christians and Muslims in the World of Islam, Princeton, Oxford, Princeton University Press.

Halbwachs Maurice, 1950, La mémoire collective, Paris, Presses Universitaires de France. HasLucK Frederick W., 1929, Christianity and Islam under the Sultans, Oxford, Clarendon Press. (rééd. Istanbul, Isis Press, 2000).

Hayden Robert M., 2002, "Competitive sharing of religious sites in South Asia and the Balkans », Current Anthropology, 43-2, pp. 205-232.

Heyberger Bernard, Madinier Rémy, (dirs.), 2010, Missions chrétiennes et islam des marges, Paris, Karthala.

Mayeur-Jaouen Catherine, 2003, « La fonction sacrale de l'image dans l'Egypte contemporaine : de l'imagerie traditionnelle à la révolution photographique ", in Heyberger B., Naef S., (dirs.), La multiplication des images en pays d'Islam, Wurzbourg, Ergon Verlag, pp. 57-80.

ReINHARD Wolfgang, 1995, "Was ist katholische Konfessionalisierung? », in Reinhard W., Schilling H., (éds.), Die katholische Konfessionalisierung, Aschendorff, Gütesloher Verlagshaus, coll. "Schriften des Vereins für Reformationsgeschichte », 198, pp. 419452.

SCARAFFIA Lucetta, 1998, Loreto, Bologne, Il Mulino.

Turner Victor, 1973, "The Center Out There: Pilgrim's Goal ", History of Religions, 12-3, pp. 191-230.

Turner Victor, Turner Édith, 1978, Image and Pilgrimage in Christian Culture, New York, Columbia University Press. 